\section{Interacción de los fármacos con los alimentos en pacientes con vejiga hiperactiva y recomendaciones para su administración}

\author{
Food interactions of the drugs \\ used in overactive bladder \\ and recommendations on their \\ administration
}

Antonio Alcántara Montero

\section{Sr. Editor:}

La vejiga hiperactiva es una alteración frecuente, cuya prevalencia varía de 7 a $27 \%$ en hombres y de 9 a $43 \%$ en mujeres. ${ }^{1}$ El tratamiento farmacológico de primera línea consiste en antimuscarínicos y mirabegrón, un agonista de los receptores $\beta 3$-adrenérgicos. ${ }^{2}$ En la práctica clínica diaria olvidamos asesorar a nuestros pacientes sobre las interacciones de los fármacos con los alimentos.

Hace poco se publicó un artículo de revisión que evaluó el efecto de los alimentos y zumos de fruta relacionados con las propiedades farmacocinéticas y terapéuticas de los medicamentos prescritos en pacientes con vejiga hiperactiva. Los autores recopilaron información en inglés de las interacciones de los medicamentos indicados para disfunción vesical en: PubMed, Embase, Cochrane y CINAHL, entre 1996 y 2015. También analizaron publicaciones electrónicas como: drugs.com, Medscape, UpToDate, Micromedex, Medical Letter y Stockley Drugs Interaction. $^{3}$

Los alimentos y el zumo de pomelo (toronja) ejercen un efecto en la farmacocinética de los medicamentos prescritos
Miembro del Grupo de Trabajo de Urología (Nefrología y Vías Urinarias) de la Sociedad Española de Médicos de Atención Primaria (SEMERGEN) en España

Correspondencia

Dr. Antonio Alcántara Montero

a.alcantara.montero@hotmail.com

Este artículo debe citarse como

Alcántara Montero A. Interacción de los fármacos con los alimentos en pacientes con vejiga hiperactiva y recomendaciones para su administración. Rev Mex Urol. 2017 mar;77(2):166-167. 
para el tratamiento de la vejiga hiperactiva. Se observó que la tolterodina y mirabegrón no ejercen efectos en los alimentos y zumos de frutas cítricas; sin embargo, darifenacina, fesoterodina, oxibutinina y solifenacina interactúan con este y otros zumos, debido a se metabolizan en el hígado por el citocromo CYP 3A4. En este caso, el zumo de toronja inhibe la acción del CYP3A4, que aumenta las concentraciones plasmáticas de dichos medicamentos, lo que resulta en riesgo de sobredosis y efectos adversos dependientes de la dosis. Los efectos de esas interacciones pueden ser potencialmente negativas para los pacientes. Además, la absorción de trospio disminuye significativamente por la acción de los alimentos. ${ }^{3}$

El Cuadro 1 muestra las principales interacciones de los fármacos con los alimentos en pacientes con vejiga hiperactiva y ofrece recomendaciones para su adecuada prescripción. ${ }^{3}$
El zumo de toronja y los alimentos pueden afectar significativamente la eficacia y seguridad de los medicamentos indicados en el tratamiento de vejiga hiperactiva. Con esta información es posible mejorar la calidad de la atención de nuestros pacientes . Hasta el momento se discuten las consecuencias de las interacciones entre alimentos y medicamentos en pacientes con vejiga hiperactiva.

\section{REFERENCIAS}

1. Gormley EA, Lightner DJ, Burgio KL, Chai TC, Clemens JQ, Culkin DJ, et al. Diagnosis and treatment of overactive bladder (Non Neurogenic) in adults AUA/SUFU Guideline. American Urological Association Education and Research, Inc. 2014. [en línea]. Dirección URL: <http://www. auanet.org/common/pdf/education/clinical-guidance/ Overactive-Bladder.pdf>. [Consulta: 28 de octubre 2016].

2. Alcántara-Montero A. Novedades en el tratamiento médico de la vejiga hiperactiva. Semergen 2015. doi: 10.1016/j. semerg.2015.10.006.

3. Paśko P, Rodacki T, Domagała-Rodacka R, Owczarek D. A short review of drug-food interactions of medicines treating overactive bladder syndrome. Int J Clin Pharm 2016. DOI:10.1007/s11096-016-0383-5.

Cuadro 1. Interacción de los fármacos con los alimentos en pacientes con vejiga hiperactiva. Recomendaciones de su administración ${ }^{3}$

\begin{tabular}{|c|c|c|}
\hline Fármaco & Interacción con los alimentos & Recomendaciones \\
\hline Darifenacina & $\begin{array}{l}\text { Interacción potencial con zumo de } \\
\text { pomelo (toronja) }\end{array}$ & $\begin{array}{l}\text { Puede indicarse con independencia de las comidas. } \\
\text { Precaución al momento de consumir zumo de pomelo (toronja) } \\
\text { durante el tratamiento. }\end{array}$ \\
\hline Fesoterodina & $\begin{array}{l}\text { Interacción potencial con zumo de } \\
\text { pomelo (toronja) }\end{array}$ & $\begin{array}{l}\text { Puede indicarse con independencia de las comidas. } \\
\text { Evitar el consumo de zumo de (toronja) pomelo durante el trata- } \\
\text { miento. }\end{array}$ \\
\hline Oxibutinina & $\begin{array}{l}\text { Interacción potencial con zumo de } \\
\text { pomelo (toronja) }\end{array}$ & $\begin{array}{l}\text { Puede indicarse con independencia de las comidas. } \\
\text { En pacientes con alteraciones de la secreción de saliva se reco- } \\
\text { mienda consumir el fármaco entre } 30 \text { minutos a } 1 \text { hora antes de } \\
\text { la comida planificada. } \\
\text { Precaución al momento de consumir zumo de pomelo (toronja) } \\
\text { durante el tratamiento. }\end{array}$ \\
\hline Solifenacina & $\begin{array}{l}\text { Interacción potencial con zumo de } \\
\text { pomelo (toronja) }\end{array}$ & $\begin{array}{l}\text { Puede indicarse con independencia de las comidas. } \\
\text { Evitar el consumo de zumo de pomelo (toronja) durante el trata- } \\
\text { miento con solifenacina. }\end{array}$ \\
\hline Tolterodina & Datos no disponibles & Puede indicarse con independencia de las comidas. \\
\hline Trospio & $\begin{array}{l}\text { Los alimentos con alto contenido } \\
\text { de grasa disminuyen la absorción } \\
\text { del fármaco }\end{array}$ & Consumirlo 1 hora antes de alguna comida o en ayuno \\
\hline Mirabegrón & Datos no disponibles & Puede indicarse con independencia de las comidas. \\
\hline
\end{tabular}

